\title{
On Degenerate Array Type Polynomials
}

\section{Lan Wu ${ }^{1}$, Xue-Yan Chen ${ }^{1}$, Muhammet Cihat Dağli ${ }^{2}$ and Feng $\mathbf{Q i}^{3,4,}$}

${ }^{1}$ Key Laboratory of Intelligent Manufacturing Technology, Inner Mongolia Minzu University, Tongliao, 028000, Inner Mongolia, China

${ }^{2}$ Department of Mathematics, Akdeniz University, Antalya, 07058, Turkey

${ }^{3}$ Institute of Mathematics, Henan Polytechnic University, Jiaozuo, 454010, China

${ }^{4}$ School of Mathematical Sciences, Tiangong University, Tianjin, 300387, China

*Corresponding Author: Feng Qi. Email: qifeng618@gmail.com

Dedicated to retired Professor Ji-Shan Tian, former vice president of Henan University, China

Received: 17 August 2021 Accepted: 27 October 2021

\section{ABSTRACT}

In the paper, with the help of the Faá di Bruno formula and an identity of the Bell polynomials of the second kind, the authors define degenerate $\lambda$-array type polynomials, establish two explicit formulas, and present several recurrence relations of degenerate $\lambda$-array type polynomials and numbers.

\section{KEYWORDS}

Degenerate array polynomial; Stirling number of the second kind; generating function; explicit formula; recurrence relation

\section{Introduction}

In this paper, we use the following notation:

$\mathbb{Z}=\{0, \pm 1, \pm 2, \ldots\}, \quad \mathbb{N}=\{1,2, \ldots\}, \quad \mathbb{N}_{0}=\{0,1,2, \ldots\}, \quad \mathbb{N}_{-}=\{-1,-2, \ldots\}$.

The Stirling numbers of the second kind $S(n, m)$ for $n \geq m \geq 0$ can be generated by

$\frac{\left(e^{t}-1\right)^{m}}{m !}=\sum_{n=m}^{\infty} S(n, m) \frac{t^{n}}{n !}$

and can be computed as

$S(n, m)=\frac{1}{m !} \sum_{i=0}^{m}(-1)^{i}\left(\begin{array}{c}m \\ i\end{array}\right)(m-i)^{n}$.

See [[1], p.206] and the paper [2].

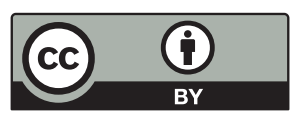

This work is licensed under a Creative Commons Attribution 4.0 International License, which permits unrestricted use, distribution, and reproduction in any medium, provided the original work is properly cited. 
The $\lambda$-array type polynomials $S(n, m ; x ; \lambda)$ were defined in [3] by the generating function

$\frac{\left(\lambda e^{t}-1\right)^{m}}{m !} e^{x t}=\sum_{n=0}^{\infty} S(n, m ; x ; \lambda) \frac{t^{n}}{n !}$.

See also the papers [4,5]. It is clear that $S(n, m ; 0 ; 1)=S(n, m)$. In the paper [6], Simsek obtained and constructed several generating functions and many relations of generalized Stirling type numbers, the array type polynomials, and Eulerian type polynomials. In the paper [7], Bayad et al. deduced interesting and meaningful identities associated with $\lambda$-array type polynomials, $\lambda$-Stirling numbers of the second kind, and the Apostol-Bernoulli numbers, while they dealt with $\lambda$-array polynomials by applying $\lambda$-delta operator. Readers interested to the Apostol-Bernoulli numbers and polynomials may consult to the papers [8-10] and closely related references therein.

In the paper [11], Carlitz introduced degenerate Bernoulli and Euler polynomials $B_{n}(x ; \gamma)$ and $E_{n}(x ; \gamma)$ by

$$
\frac{t}{(1+\gamma t)^{1 / \gamma}-1}(1+\gamma t)^{x / \gamma}=\sum_{n=0}^{\infty} B_{n}(x ; \gamma) \frac{t^{n}}{n !}
$$

and

$$
\frac{2}{(1+\gamma t)^{1 / \gamma}+1}(1+\gamma t)^{x / \gamma}=\sum_{n=0}^{\infty} E_{n}(x ; \gamma) \frac{t^{n}}{n !}
$$

respectively. For $x=0$, the quantities $B_{n}(0 ; \gamma)$ and $E_{n}(0 ; \gamma)$ are called as degenerate Bernoulli and Euler numbers. Since $\lim _{\gamma \rightarrow 0}(1+\gamma t)^{1 / \gamma}=e^{t}$, Eqs. (3) and (4) reduce to the generating functions for classical Bernoulli and Euler polynomials, respectively.

We now define degenerate $\lambda$-array type polynomials $S(n, m ; x ; \lambda ; \gamma)$ by

$$
\frac{\left[\lambda(1+\gamma t)^{1 / \gamma}-1\right]^{m}}{m !}(1+\gamma t)^{x / \gamma}=\sum_{n=0}^{\infty} S(n, m ; x ; \lambda ; \gamma) \frac{t^{n}}{n !} .
$$

It is easy to see that

$$
\lim _{\gamma \rightarrow 0} S(n, m ; x ; \lambda ; \gamma)=S(n, m ; x ; \lambda)
$$

which is defined by (2). When $x=0$, we call the quantities $S(n, m ; 0 ; \lambda ; \gamma)$ degenerate $\lambda$-array type numbers.

In this paper, utilizing the Faá di Bruno formula and an identity of the Bell polynomials of the second kind, we establish several explicit formulas and recurrence relations of (degenerate) $\lambda$-array type numbers and polynomials.

Let us notice that the Faá di Bruno formula, which can be viewed as an extension of chain rule to higher derivatives, has been applied to establish explicit and closed-form formulas of many important numbers and polynomials in analytic and combinatorial number theory. For more details, please refer to, for example, the papers [12-18] and closely related references therein. 


\section{Some Identities of the Bell Polynomials of the Second Kind}

The Bell polynomials of the second kind $B_{n, k}\left(x_{1}, x_{2}, \ldots, x_{n-k+1}\right)$ for $n \geq k \geq 0$ can be defined by

$$
\begin{aligned}
B_{n, k}\left(x_{1}, x_{2}, \ldots, x_{n-k+1}\right)= & \sum_{\substack{1 \leq i \leq n-k+1 \\
\ell_{i} \in\{0\} \cup \mathbb{N} \\
\sum_{i=1}^{n-k+1} i \ell_{i}=n\\
}} \frac{n !}{\prod_{i=1}^{n-k+1} \ell_{i} !} \prod_{i=1}^{n-k+1} \ell_{i}=k
\end{aligned}
$$

See [[1], p. 134]. For $n \in \mathbb{N}$, the Faà di Bruno formula is described in [[1], p.139] in terms of the Bell polynomials of the second kind $B_{n, k}\left(x_{1}, x_{2}, \ldots, x_{n-k+1}\right)$ by

$\frac{d^{n}}{d t^{n}} f \circ h(t)=\sum_{k=1}^{n} f^{(k)}(h(t)) B_{n, k}\left(h^{\prime}(t), h^{\prime \prime}(t), \ldots, h^{(n-k+1)}(t)\right)$.

The formula

$B_{n, k}\left(1,1-\lambda,(1-\lambda)(1-2 \lambda), \ldots, \prod_{\ell=0}^{n-k}(1-\ell \lambda)\right)=\frac{(-1)^{k}}{k !} \sum_{\ell=0}^{k}(-1)^{\ell}\left(\begin{array}{l}k \\ \ell\end{array}\right) \prod_{q=0}^{n-1}(\ell-q \lambda)$

has been applied and reviewed in [[16], Lemma 2.2], [[17], Remark 6.1], and [[19], Section 1.3]. The explicit formula (8) is equivalent to

$$
B_{n, k}\left(\langle\lambda\rangle_{1},\langle\lambda\rangle_{2}, \ldots,\langle\lambda\rangle_{n-k+1}\right)=\frac{(-1)^{k}}{k !} \sum_{\ell=0}^{k}(-1)^{\ell}\left(\begin{array}{l}
k \\
\ell
\end{array}\right)\langle\lambda \ell\rangle_{n},
$$

which was presented in [[20], Theorems 2.1 and 4.1], where the falling factorial $\langle x\rangle_{n}$ is defined for $x \in \mathbb{C}$ by

$$
\langle x\rangle_{n}=\prod_{k=0}^{n-1}(x-k)= \begin{cases}x(x-1) \ldots m(x-n+1), & n \in \mathbb{N} \\ 1, & n=0 .\end{cases}
$$

When $n \in \mathbb{N}$, the explicit formulas (8) and (9) can be rearranged as

$$
B_{n, k}\left(1,1-\lambda,(1-\lambda)(1-2 \lambda), \ldots, \prod_{\ell=0}^{n-k}(1-\ell \lambda)\right)= \begin{cases}(-1)^{k} \frac{\lambda^{n} n !}{k !} \sum_{\ell=0}^{k}(-1)^{\ell}\left(\begin{array}{c}
k \\
\ell
\end{array}\right)\left(\begin{array}{c}
\ell / \lambda \\
n
\end{array}\right), & \lambda \neq 0 \\
S(n, k), & \lambda=0\end{cases}
$$


where extended binomial coefficient $\left(\begin{array}{l}z \\ w\end{array}\right)$ is defined by

$$
\left(\begin{array}{l}
z \\
w
\end{array}\right)= \begin{cases}\frac{\Gamma(z+1)}{\Gamma(w+1) \Gamma(z-w+1)}, & z \notin \mathbb{N}_{-}, \quad w, z-w \notin \mathbb{N}_{-} \\
0, & z \notin \mathbb{N}_{-}, \quad w \in \mathbb{N}_{-} \text {or } z-w \in \mathbb{N}_{-} \\
\frac{\langle z\rangle_{w}}{w !}, & z \in \mathbb{N}_{-}, \quad w \in \mathbb{N}_{0} \\
\frac{\langle z\rangle_{z-w}}{(z-w) !}, & z, w \in \mathbb{N}_{-}, \quad z-w \in \mathbb{N}_{0} \\
0, & z, w \in \mathbb{N}_{-}, \quad z-w \in \mathbb{N}_{-} \\
\infty, & z \in \mathbb{N}_{-}, \quad w \notin \mathbb{Z}\end{cases}
$$

and the classical Euler gamma function $\Gamma(z)$ can be defined by

$\Gamma(z)=\lim _{n \rightarrow \infty} \frac{n ! n^{z}}{\prod_{k=0}^{n}(z+k)}, \quad z \in \mathbb{C} \backslash\{0,-1,-2, \ldots\}$.

For new results and applications about the Bell polynomials' of the second kind $B_{n, k}$, please refer to the papers $[13,19,21-23]$ and closely related references therein.

\section{Explicit Formulas of Degenerate $\lambda$-Array Type Polynomials}

In this section, we establish two explicit formulas for degenerate $\lambda$-array type numbers and polynomials, respectively.

Theorem 3.1. For $n \in \mathbb{N}$, degenerate $\lambda$-array type numbers $S(n, m ; 0 ; \lambda ; \gamma)$ can be computed by

$S(n, m ; 0 ; \lambda ; \gamma)=\frac{n !}{m !} \lambda^{n}(\lambda-1)^{m} \sum_{k=1}^{n} \frac{\langle m\rangle_{k}}{k !} \frac{1}{(1 / \lambda-1)^{k}} \sum_{\ell=0}^{k}(-1)^{\ell}\left(\begin{array}{c}k \\ \ell\end{array}\right)\left(\begin{array}{c}\ell / \lambda \\ n\end{array}\right), \quad \lambda \neq 0$.

Proof. Making use of $f(u)=(\lambda u-1)^{m}$ and $u=h(t)=(1+\gamma t)^{1 / \gamma} \rightarrow 1$ as $t \rightarrow 0$ in the Faá di Bruno formula (6) and applying (10) result in

$$
\begin{aligned}
\frac{d^{n}\left(\left[\lambda(1+\gamma t)^{1 / \gamma}-1\right]^{m}\right)}{d t^{n}}= & \sum_{k=1}^{n} \frac{d^{k}\left[(\lambda u-1)^{m}\right]}{d u^{k}} B_{n, k}\left(h^{\prime}(t), h^{\prime \prime}(t), \ldots, h^{(k-\ell+1)}(t)\right) \\
= & \sum_{k=1}^{n}\langle m\rangle_{k}(\lambda u-1)^{m-k} \lambda^{k} B_{n, k}\left((1+\gamma t)^{1 / \gamma-1},(1-\gamma)(1+\gamma t)^{1 / \gamma-2},\right. \\
& \left.\ldots,(1-\gamma)(1-2 \gamma) \ldots[1-(n-k) \gamma](1+\gamma t)^{1 / \gamma-(n-k+1)}\right) \\
\rightarrow & \sum_{k=1}^{n}\langle m\rangle_{k}(\lambda-1)^{m-k} \lambda^{k} B_{n, k}\left(1,1-\gamma, \ldots, \prod_{\ell=0}^{n-k}(1-\ell \gamma)\right) \\
= & \sum_{k=1}^{n}\langle m\rangle_{k}(\lambda-1)^{m-k} \lambda^{k}(-1)^{k} \frac{\lambda^{n} n !}{k !} \sum_{\ell=0}^{k}(-1)^{\ell}\left(\begin{array}{c}
k \\
\ell
\end{array}\right)\left(\begin{array}{c}
\ell / \lambda \\
n
\end{array}\right)
\end{aligned}
$$




$$
=n ! \lambda^{n}(\lambda-1)^{m} \sum_{k=1}^{n} \frac{\langle m\rangle_{k}}{k !} \frac{1}{(1 / \lambda-1)^{k}} \sum_{\ell=0}^{k}(-1)^{\ell}\left(\begin{array}{c}
k \\
\ell
\end{array}\right)\left(\begin{array}{c}
\ell / \lambda \\
n
\end{array}\right)
$$

as $t \rightarrow 0$. Considering the generating function in (5) for $x=0$, we proved the explicit formula (12). The proof of Theorem 3.1 is complete.

Remark 3.1. From (5), it follows immediately that $S(0, m ; 0 ; \lambda ; \gamma)=\frac{(\lambda-1)^{m}}{m !}$.

By virtue of the explicit formula (12), we obtain the first few values of degenerate $\lambda$-array type numbers $S(n, m ; 0 ; \lambda ; \gamma)$ for $1 \leq n \leq 6$ as follows:

$$
\begin{aligned}
& S(1, m ; 0 ; \lambda ; \gamma)=\frac{1}{(m-1) !} \lambda(\lambda-1)^{m-1}, \\
& S(2, m ; 0 ; \lambda ; \gamma)=\frac{1}{(m-1) !} \lambda(\lambda-1)^{m-2}[\gamma(1-\lambda)+\lambda m-1], \\
& S(3, m ; 0 ; \lambda ; \gamma)=\frac{1}{(m-1) !} \lambda(\lambda-1)^{m-3}\left[2 \gamma^{2}(1-\lambda)^{2}+3 \gamma(1-\lambda)(\lambda m-1)+\lambda^{2} m^{2}-3 \lambda m+\lambda+1\right] \text {, } \\
& S(4, m ; 0 ; \lambda ; \gamma)=\frac{\lambda(\lambda-1)^{m-4}}{(m-1) !}\left[6 \gamma^{3}(1-\lambda)^{3}+11 \gamma^{2}(1-\lambda)^{2}(\lambda m-1)\right. \\
& \left.+6 \gamma(1-\lambda)\left(\lambda^{2} m^{2}-3 \lambda m+\lambda+1\right)+\lambda^{3} m^{3}-\lambda^{2}\left(6 m^{2}-4 m+1\right)+\lambda(7 m-4)-1\right], \\
& S(5, m ; 0 ; \lambda ; \gamma)=\frac{\lambda(\lambda-1)^{m-5}}{(m-1) !}\left\{24 \gamma^{4}(\lambda-1)^{4}-50 \gamma^{3}(\lambda-1)^{3}(\lambda m-1)\right. \\
& +35 \gamma^{2}(\lambda-1)^{2}\left(\lambda^{2} m^{2}-3 \lambda m+\lambda+1\right) \\
& -10 \gamma(\lambda-1)\left[\lambda^{3} m^{3}-\lambda^{2}\left(6 m^{2}-4 m+1\right)+\lambda(7 m-4)-1\right] \\
& \left.+\lambda^{4} m^{4}-\lambda^{3}\left(10 m^{3}-10 m^{2}+5 m-1\right)+\lambda^{2}\left(25 m^{2}-30 m+11\right)-\lambda(15 m-11)+1\right\}, \\
& S(6, m ; 0 ; \lambda ; \gamma)=-\frac{\lambda(\lambda-1)^{m-6}}{(m-1) !}\left\{120 \gamma^{5}(\lambda-1)^{5}-274 \gamma^{4}(\lambda-1)^{4}(\lambda m-1)\right. \\
& +225 \gamma^{3}(\lambda-1)^{3}\left(\lambda^{2} m^{2}-3 \lambda m+\lambda+1\right) \\
& -85 \gamma^{2}(\lambda-1)^{2}\left[\lambda^{3} m^{3}-\lambda^{2}\left(6 m^{2}-4 m+1\right)+\lambda(7 m-4)-1\right] \\
& +15 \gamma(\lambda-1)\left[\lambda^{4} m^{4}-\lambda^{3}\left(10 m^{3}-10 m^{2}+5 m-1\right)\right. \\
& \left.+\lambda^{2}\left(25 m^{2}-30 m+11\right)-\lambda(15 m-11)+1\right]-\lambda^{5} m^{5} \\
& +\lambda^{4}\left(15 m^{4}-20 m^{3}+15 m^{2}-6 m+1\right)-\lambda^{3}\left(65 m^{3}-120 m^{2}+91 m-26\right) \\
& \left.+2 \lambda^{2}\left(45 m^{2}-73 m+33\right)-\lambda(31 m-26)+1\right\} \text {. }
\end{aligned}
$$


Remark 3.2. The explicit formula (12) in Theorem 3.1 and seven concrete values listed in Remark 3.1 reveal that degenerate $\lambda$-array type numbers $S(n, m ; 0 ; \lambda ; \gamma)$ are polynomials of $\lambda$ and $\gamma$ with degrees $m$ and $n-1 \geq 0$, respectively.

Theorem 3.2. For $n \in \mathbb{N}$, degenerate $\lambda$-array type polynomials $S(n, m ; x ; \lambda ; \gamma)$ can be computed by

$S(n, m ; x ; \lambda ; \gamma)=\frac{n !}{m !} \lambda^{n}(\lambda-1)^{m} \sum_{k=1}^{n} \frac{(-1)^{k}}{k !}\left[\sum_{\ell=0}^{k}\left(\begin{array}{l}k \\ \ell\end{array}\right) \frac{\langle m\rangle_{\ell}\langle x\rangle_{k-\ell}}{(1-1 / \lambda)^{\ell}}\right]\left[\sum_{\ell=0}^{k}(-1)^{\ell}\left(\begin{array}{c}k \\ \ell\end{array}\right)\left(\begin{array}{c}\ell / \lambda \\ n\end{array}\right)\right]$.

Proof. For $k \in \mathbb{N}$, it is easy to see that

$$
\begin{aligned}
\frac{d^{k}}{d u^{k}}\left[\frac{(\lambda u-1)^{m}}{m !} u^{x}\right] & =\frac{1}{m !} \sum_{\ell=0}^{k}\left(\begin{array}{l}
k \\
\ell
\end{array}\right)\langle m\rangle_{\ell} \lambda^{\ell}(\lambda u-1)^{m-\ell}\langle x\rangle_{k-\ell} u^{x-k+\ell} \\
& \rightarrow \frac{1}{m !} \sum_{\ell=0}^{k}\left(\begin{array}{l}
k \\
\ell
\end{array}\right)\langle m\rangle_{\ell}\langle x\rangle_{k-\ell} \lambda^{\ell}(\lambda-1)^{m-\ell}
\end{aligned}
$$

as $u \rightarrow 1$. Letting $u=h(t)=(1+\gamma t)^{1 / \gamma} \rightarrow 1$ as $t \rightarrow 0$ and making use of the Faà di Bruno formula (7) give

$$
\begin{aligned}
& \frac{d^{n}}{d t^{n}}\left(\frac{\left[\lambda(1+\gamma t)^{1 / \gamma}-1\right]^{m}}{m !}(1+\gamma t)^{x / \gamma}\right) \\
= & \sum_{k=1}^{n} \frac{d^{k}}{d u^{k}}\left[\frac{(\lambda u-1)^{m}}{m !} u^{x}\right] B_{n, k}\left(h^{\prime}(t), h^{\prime \prime}(t), \ldots, h^{(k-\ell+1)}(t)\right) \\
= & \frac{1}{m !} \sum_{k=1}^{n}\left[\sum_{\ell=0}^{k}\left(\begin{array}{l}
k \\
\ell
\end{array}\right)\langle m\rangle_{\ell} \lambda^{\ell}(\lambda u-1)^{m-\ell}\langle x\rangle_{k-\ell} u^{x-k+\ell}\right] \\
& \times B_{n, k}\left((1+\gamma t)^{1 / \gamma-1},(1-\gamma)(1+\gamma t)^{1 / \gamma-2}, \ldots,(1+\gamma t)^{1 / \gamma-(n-k+1)} \prod_{\ell=0}^{n-k}(1-\ell \gamma)\right) \\
\rightarrow & \frac{1}{m !} \sum_{k=1}^{n}\left[\sum_{\ell=0}^{k}\left(\begin{array}{l}
k \\
\ell
\end{array}\right)\langle m\rangle_{\ell}\langle x\rangle_{k-\ell} \lambda^{\ell}(\lambda-1)^{m-\ell}\right] B_{n, k}\left(1,1-\gamma, \ldots, \prod_{\ell=0}^{n-k}(1-\ell \gamma)\right) \\
= & \frac{1}{m !} \sum_{k=1}^{n}\left[\sum_{\ell=0}^{k}\left(\begin{array}{l}
k \\
\ell
\end{array}\right)\langle m\rangle_{\ell}\langle x\rangle_{k-\ell} \lambda^{\ell}(\lambda-1)^{m-\ell}\right]\left[(-1)^{k} \frac{\lambda^{n} n !}{k !} \sum_{\ell=0}^{k}(-1)^{\ell}\left(\begin{array}{c}
k \\
\ell
\end{array}\right)\left(\begin{array}{c}
\ell / \lambda \\
n
\end{array}\right)\right] \\
= & \frac{n !}{m !} \lambda^{n}(\lambda-1)^{m} \sum_{k=1}^{n} \frac{(-1)^{k}}{k !}\left[\sum_{\ell=0}^{k}\left(\begin{array}{c}
k \\
\ell
\end{array}\right) \frac{\langle m\rangle_{\ell}\langle x\rangle_{k-\ell}}{(1-1 / \lambda)^{\ell}}\right]\left[\sum_{\ell=0}^{k}(-1)^{\ell}\left(\begin{array}{c}
k \\
\ell
\end{array}\right)\left(\begin{array}{c}
\ell / \lambda \\
n
\end{array}\right)\right]
\end{aligned}
$$

as $t \rightarrow 0$. Considering the generating function in (5), we proved the explicit formula (13). The proof of Theorem 3.2 is complete. 
Remark 3.3. From the generating function (5), we can easily obtain $S(0, m ; x ; \lambda ; \gamma)=\frac{(\lambda-1)^{m}}{m !}$.

By virtue of the explicit formula (13), we can calculate the first few values of degenerate $\lambda$-array type polynomials $S(n, m ; x ; \lambda ; \gamma)$ for $1 \leq n \leq 3$ as follows:

$$
\begin{aligned}
S(1, m ; x ; \lambda ; \gamma)= & \frac{(\lambda-1)^{m-1}}{m !}[\lambda(m+x)-x], \\
S(2, m ; x ; \lambda ; \gamma)= & \frac{(\lambda-1)^{m-2}}{m !}\left[\lambda^{2}(m+x)(m-\gamma+x)+\lambda m(\gamma-2 x-1)+2 \lambda x(\gamma-x)+x(x-\gamma)\right], \\
S(3, m ; x ; \lambda ; \gamma)= & \frac{(\lambda-1)^{m-3}}{m !}\left\{\lambda^{3}(m+x)\left[2 \gamma^{2}-3 \gamma(m+x)+(m+x)^{2}\right]\right. \\
& +\lambda^{2}\left[3 m^{2}(\gamma-x-1)-m\left(4 \gamma^{2}-12 \gamma x-3 \gamma+6 x^{2}+3 x-1\right)-3 x\left(2 \gamma^{2}-3 \gamma x+x^{2}\right)\right] \\
& \left.+\lambda\left[m\left(2 \gamma^{2}-6 \gamma x-3 \gamma+3 x^{2}+3 x+1\right)+3 x\left(2 \gamma^{2}-3 \gamma x+x^{2}\right)\right]-x\left(2 \gamma^{2}-3 \gamma x+x^{2}\right)\right\} .
\end{aligned}
$$

Remark 3.4. From the explicit formula (13) in Theorem 3.2 and the four concrete values in Remark 3.3, we conclude that degenerate $\lambda$-array type polynomials $S(n, m ; x ; \lambda ; \gamma)$ are polynomials of $x, \lambda$, and $\gamma$ of degrees $n, m$, and $n-1 \geq 0$, respectively.

Remark 3.5. When $x=0$ in Theorem 3.2, the explicit formula (13) becomes (12) in Theorem 3.1 .

Remark 3.6. For further better understanding degenerate $\lambda$-array type polynomials $S(n, m ; x ; \lambda ; \gamma)$, we demonstrate two angles of the graph of $S(3,2 ; 4 ; \lambda ; \gamma)$ for $-9<\lambda<9$ and $0<\gamma<9$ in Fig. 1. The blue plane in Fig. 1 is the $(\lambda, \gamma)$-plane.
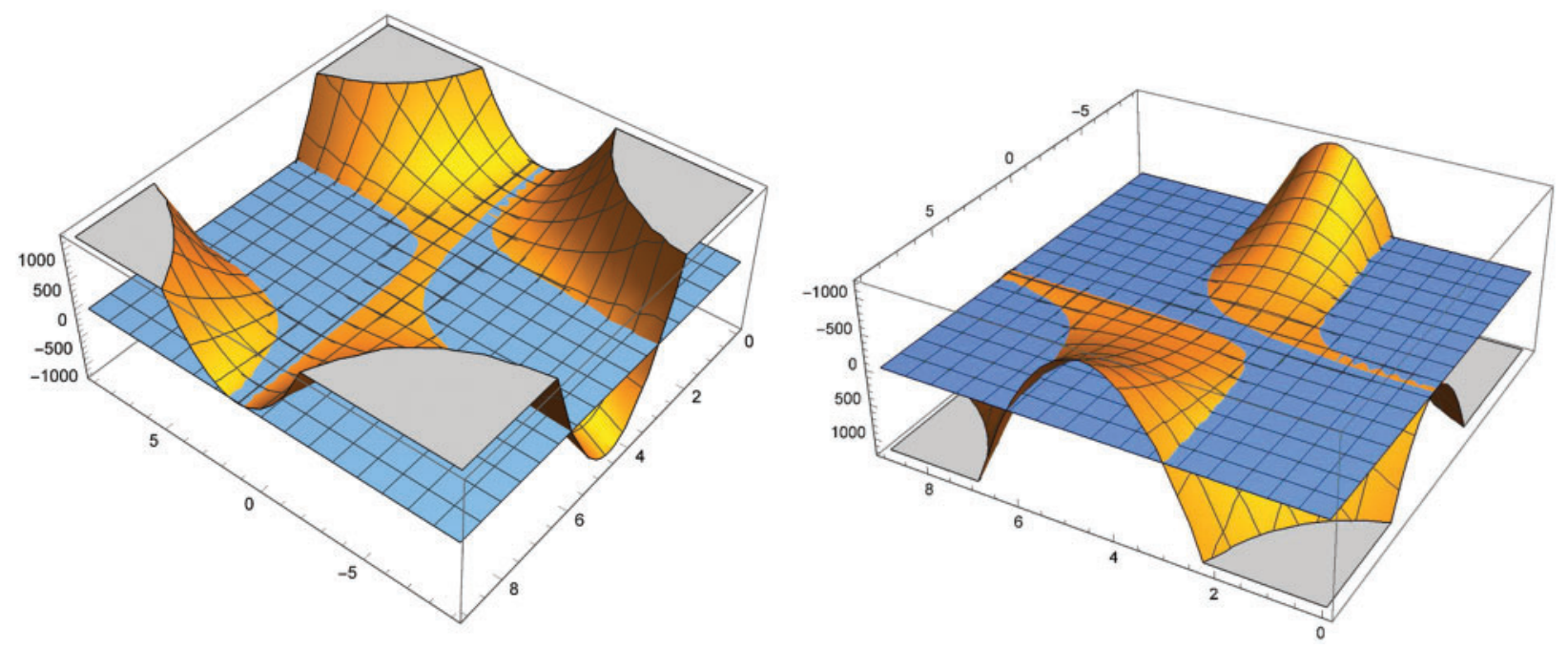

Figure 1: Two angles of the graph of $S(3,2 ; 4 ; \lambda ; \gamma)$ for $-9<\lambda<9$ and $0<\gamma<9$, plotted by Wolfram Mathematica 12.0 
Remark 3.7. For further better understanding degenerate $\lambda$-array type polynomials $S(n, m ; x ; \lambda ; \gamma)$, we demonstrate two angles of the graph of $S(3,2 ; x ; 4 ; \gamma)$ for $0<x<9$ and $0<\gamma<9$ in Fig. 2. The blue plane in Fig. 2 is the $(x, \gamma)$-plane.
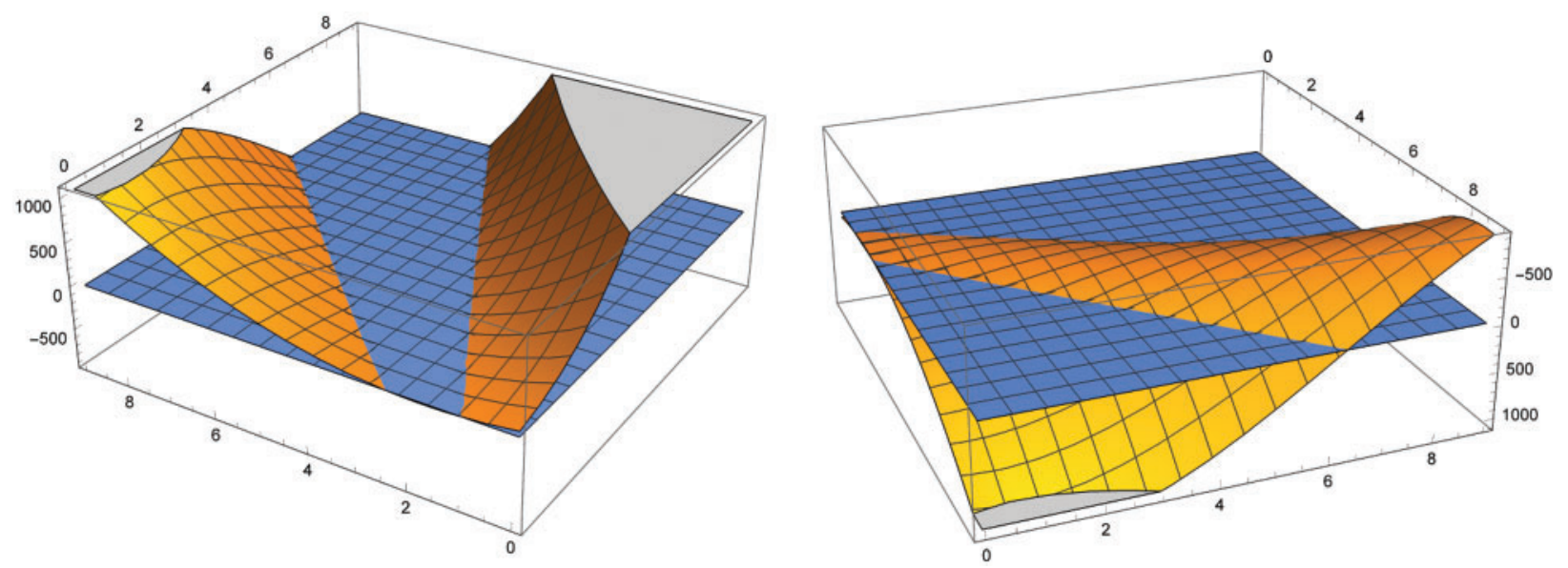

Figure 2: Two angles of the graph of $S(3,2 ; x ; 4 ; \gamma)$ for $0<x<9$ and $0<\gamma<9$, plotted by Wolfram Mathematica 12.0

Remark 3.8. For further better understanding degenerate $\lambda$-array type polynomials $S(n, m ; x ; \lambda ; \gamma)$, we demonstrate two angles of the graph of $S(3,2 ; x ; \lambda ; 4)$ for $0<x<9$ and $-9<\lambda<9$ in Fig. 3. The blue plane in Fig. 3 is the $(x, \lambda)$-plane.
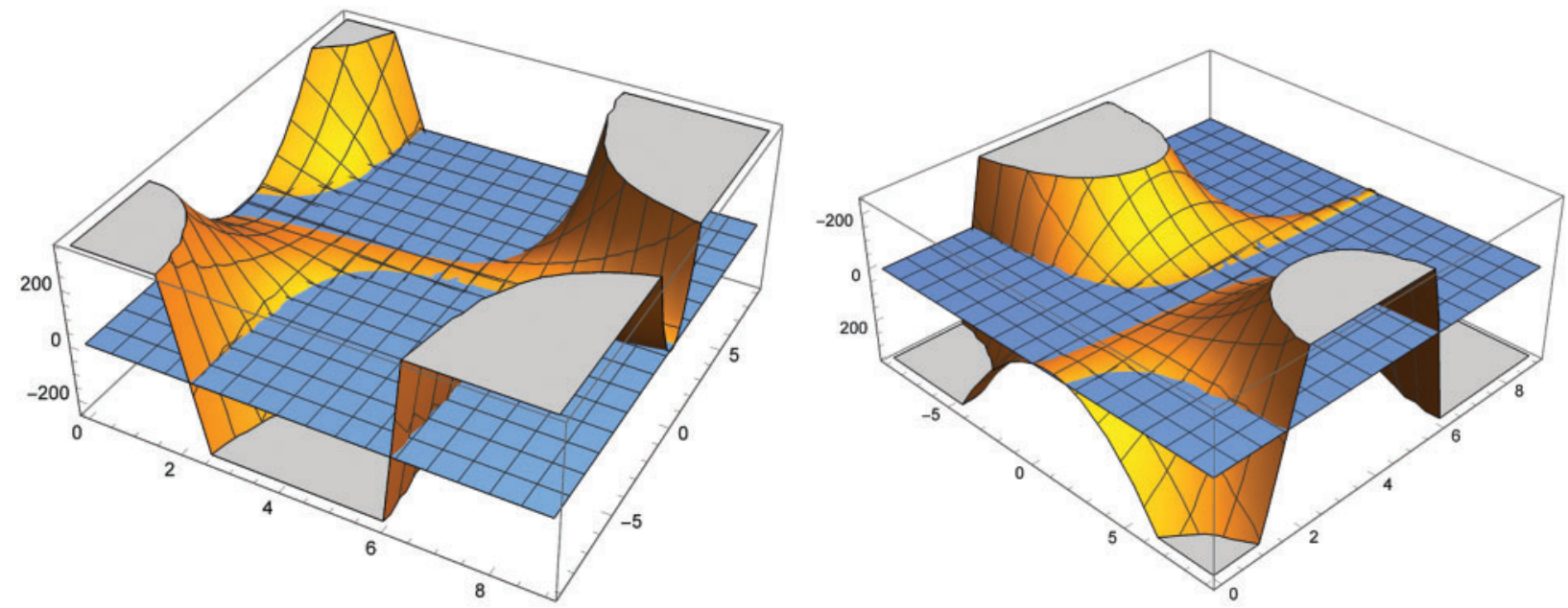

Figure 3: Two angles of the graph of $S(3,2 ; x ; \lambda ; 4)$ for $0<x<9$ and $-9<\lambda<9$, plotted by Wolfram Mathematica 12.0

\section{Recurrence Relations of Degenerate $\lambda$-Array Type Polynomials}

In this section, we establish several recurrence relations of degenerate $\lambda$-array type polynomials. 
Theorem 4.1. Degenerate $\lambda$-array type polynomials satisfy the recurrence relation $\lambda S(n, m ; x+1 ; \lambda ; \gamma)=(m+1) S(n, m+1 ; x ; \lambda ; \gamma)+S(n, m ; x ; \lambda ; \gamma)$.

Proof. From Eq. (5), it follows that

$$
\begin{aligned}
& \sum_{n=0}^{\infty}[S(n, m ; x+1 ; \lambda ; \gamma)-S(n, m ; x ; \lambda ; \gamma)] \frac{t^{n}}{n !} \\
= & \frac{\left[\lambda(1+\gamma t)^{1 / \gamma}-1\right]^{m}}{m !}(1+\gamma t)^{x / \gamma}\left[(1+\gamma t)^{1 / \gamma}-1\right] \\
= & \frac{\left[\lambda(1+\gamma t)^{1 / \gamma}-1\right]^{m+1}}{m !}(1+\gamma t)^{x / \gamma}\left[\frac{(1+\gamma)^{1 / \gamma}-1}{\lambda(1+\gamma t)^{1 / \gamma}-1}\right] \\
= & \frac{\left[\lambda(1+\gamma t)^{1 / \gamma}-1\right]^{m+1}}{m !}(1+\gamma t)^{x / \gamma}\left[\frac{1}{\lambda}+\frac{(1-\lambda) / \lambda}{\lambda(1+\gamma t)^{1 / \gamma}-1}\right] \\
= & \frac{m+1}{\lambda} \frac{\left[\lambda(1+\gamma t)^{1 / \gamma}-1\right]^{m+1}}{(m+1) !}(1+\gamma t)^{x / \gamma}+\frac{1-\lambda}{\lambda} \frac{\left[\lambda(1+\gamma t)^{1 / \gamma}-1\right]^{m}}{m !}(1+\gamma t)^{x / \gamma} \\
= & \frac{m+1}{\lambda} \sum_{n=0}^{\infty} S(n, m+1 ; x ; \lambda ; \gamma) \frac{t^{n}}{n !}+\frac{1-\lambda}{\lambda} \sum_{n=0}^{\infty} S(n, m ; x ; \lambda ; \gamma) \frac{t^{n}}{n !} .
\end{aligned}
$$

Comparing coefficients of the terms $\frac{t^{n}}{n !}$ on both sides concludes (14). The proof of Theorem 4.1 is complete.

Theorem 4.2. Degenerate $\lambda$-array type polynomials $S(n, m ; x ; \lambda ; \gamma)$ satisfy the recurrence relation

$S(n+1, m ; y+\gamma ; \lambda ; \gamma)=\lambda S(n, m-1 ; y+1 ; \lambda ; \gamma)+(y+\gamma) S(n, m ; y ; \lambda ; \gamma)$.

Consequently, we have

$S(n+1, m ; y ; \lambda)=\lambda S(n, m-1 ; y+1 ; \lambda)+y S_{2}(n, m ; y ; \lambda)$.

Proof. Differentiating with respect to $t$ yields

$$
\begin{aligned}
\frac{d}{d t}\left\{\left[\lambda(1+\gamma t)^{1 / \gamma}-1\right]^{m}(1+\gamma t)^{x / \gamma}\right\}= & \lambda m\left[\lambda(1+\gamma t)^{1 / \gamma}-1\right]^{m-1}(1+\gamma t)^{(x+1-\gamma) / \gamma} \\
& +x\left[\lambda(1+\gamma t)^{1 / \gamma}-1\right]^{m}(1+\gamma t)^{(x-\gamma) / \gamma}
\end{aligned}
$$

where, on the other hand,

$$
\begin{aligned}
\frac{d}{d t}\left\{\left[\lambda(1+\gamma t)^{1 / \gamma}-1\right]^{m}(1+\gamma t)^{x / \gamma}\right\} & =m ! \sum_{n=0}^{\infty} S(n+1, m ; x ; \lambda ; \gamma) \frac{t^{n}}{n !}, \\
x\left[\lambda(1+\gamma t)^{1 / \gamma}-1\right]^{m}(1+\gamma t)^{(x-\gamma) / \gamma} & =x m ! \sum_{n=0}^{\infty} S(n, m ; x-\gamma ; \lambda ; \gamma) \frac{t^{n}}{n !},
\end{aligned}
$$


and

$\lambda m\left[\lambda(1+\gamma t)^{1 / \gamma}-1\right]^{m-1}(1+\gamma t)^{(x+1-\gamma) / \gamma}=\lambda m ! \sum_{n=0}^{\infty} S(n, m-1 ; x+1-\gamma ; \lambda ; \gamma) \frac{t^{n}}{n !}$.

Further replacing $x$ by $y+\gamma$ and simplifying lead to (15).

Taking $\gamma \rightarrow 0$ in (15) and considering (6) give (16). The proof of Theorem 4.2 is complete.

Remark 4.1. One of anonymous referees commented that the $\lambda$-array type polynomials are related to numbers considered in the papers [24-26].

\section{Conclusions}

In this paper, with the help of the Faá di Bruno formula (7) and the identity (10) for the Bell polynomials of the second kind $B_{n, k}$, we define degenerate $\lambda$-array type polynomials $S(n, m ; x ; \lambda ; \gamma)$ by (5), establish two explicit formulas (12) and (13) in Theorems 3.1 and 3.2, and present several recurrence relations (14), (15), and (16) of degenerate $\lambda$-array type polynomials and numbers $S(n, m ; x ; \lambda ; \gamma)$ and $S(n, m ; \lambda ; \gamma)$ in Theorems 4.1 and 4.2 .

Acknowledgement: The authors thank the editors and anonymous referees for their careful corrections to, valuable comments on, and helpful suggestions to the original version of this paper.

Funding Statement: The first two authors, Mrs. Lan Wu and Xue-Yan Chen, were partially supported by the College Scientific Research Project of Inner Mongolia (Grant No. NJZY19156 and Grant No. NJZZ19144), by the Natural Science Foundation Project of Inner Mongolia (Grant No. 2021LHMS05030), and by the Development Plan for Young Technological Talents in Colleges and Universities of Inner Mongolia (Grant No. NJYT22051) in China.

Conflicts of Interest: The authors declare that they have no conflicts of interest to report regarding the present study.

\section{References}

1. Comtet, L. (1974). Advanced combinatorics: The art of finite and infinite expansions. Revised and Enlarged Edition, D. Dordrecht: Reidel Publishing Co.

2. Qi, F. (2016). Diagonal recurrence relations, inequalities, and monotonicity related to the Stirling numbers of the second kind. Mathematical Inequalities and Applications, 19(1), 313-323. DOI 10.7153/mia-19-23.

3. Caki, N. P., Milovanovi, G. V. (2004). On generalized Stirling numbers and polynomials. Math Balkanica, 18(3-4), 241-248.

4. Chang, C. H., Ha, C. W. (2006). A multiplication theorem for the Lerch zeta function and explicit representations of the Bernoulli and Euler polynomials. Journal of Mathematical Analysis and Applications, 315(2), 758-767. DOI 10.1016/j.jmaa.2005.08.013.

5. Simsek, Y. (2012). Interpolation function of generalized q-bernstein-type basis polynomials and applications. In: Curves and surfaces, pp. 647-662. Heidelberg: Springer.

6. Simsek, Y. (2013). Generating functions for generalized Stirling type numbers, array type polynomials, Eulerian type polynomials and their applications. Fixed Point Theory and Applications, 2013(1), 1-28. DOI 10.1186/1687-1812-2013-87.

7. Bayad, A., Simsek, Y., Srivastava, H. M. (2014). Some array type polynomials associated with special numbers and polynomials. Applied Mathematics and Computation, 244, 149-157. DOI 10.1016/j.amc.2014.06.086. 
8. He, Y., Araci, S., Srivastava, H. M. (2016). Some new formulas for the products of the Apostol type polynomials. Advances in Difference Equations, 2016(1), 1-18. DOI 10.1186/s13662-016-1014-0.

9. He, Y., Araci, S., Srivastava, H. M., Acikgoz, M. (2015). Some new identities for the Apostol-Bernoulli polynomials and the Apostol-Genocchi polynomials. Applied Mathematics and Computation, 262, 31-41. DOI 10.1016/j.amc.2015.03.132.

10. Kilar, N., Simsek, Y. (2019). Identities and relations for Fubini type numbers and polynomials via generating functions and $p$-adic integral approach. Publications de L'Institut Mathematique, 106(120), 113-123. DOI 10.2298/PIM1920113K.

11. Carlitz, L. (1979). Degenerate Stirling Bernoulli and Eulerian numbers. Utilitas Mathematica, 15, 51-88.

12. Dağl1, M. C. (2021). A new recursive formula arising from a determinantal expression for weighted Delannoy numbers. Turkish Journal of Mathematics, 45(1), 471-478. DOI 10.3906/mat-2009-92.

13. Dağlı, M. C. (2021). Closed formulas and determinantal expressions for higher-order Bernoulli and Euler polynomials in terms of Stirling numbers. Revista de la Real Academia de Ciencias Exactas Físicas y Naturales Serie A Matemáticas, 115(1), 1-8.

14. Dai, L., Pan, H. (2020). Closed forms for degenerate Bernoulli polynomials. Bulletin of the Australian Mathematical Society, 101(2), 207-217. DOI 10.1017/S0004972719001266.

15. Hu, S., Kim, M. S. (2018). Two closed forms for the Apostol-Bernoulli polynomials. The Ramanujan Journal, 46(1), 103-117. DOI 10.1007/s11139-017-9907-4.

16. Qi, F., Čerňanová, V., Shi, X. T., Guo, B. N. (2018). Some properties of central Delannoy numbers. Journal of Computational and Applied Mathematics, 328, 101-115. DOI 10.1016/j.cam.2017.07.013.

17. Qi, F., Guo, B. N. (2017). Explicit formulas for special values of the Bell polynomials of the second kind and for the Euler numbers and polynomials. Mediterranean Journal of Mathematics, 14(3), 1-14. DOI 10.1007/s00009-017-0939-1.

18. Qi, F., Guo, B. N. (2016). Some determinantal expressions and recurrence relations of the Bernoulli polynomials. Mathematics, 4(4), 65. DOI 10.3390/math4040065.

19. Qi, F., Niu, D. W., Lim, D., Yao, Y. H. (2020). Special values of the Bell polynomials of the second kind for some sequences and functions. Journal of Mathematical Analysis and Applications, 491(2), 124382. DOI 10.1016/j.jmaa.2020.124382.

20. Qi, F., Niu, D. W., Lim, D., Guo, B. N. (2020). Closed formulas and identities for the Bell polynomials and falling factorials. Contributions to Discrete Mathematics, 15(1), 163-174. DOI 10.11575/cdm.v15i1.68111.

21. Guo, B. N., Lim, D., Qi, F. (2021). Series expansions of powers of arcsine, closed forms for special values of Bell polynomials, and series representations of generalized logsine functions. AIMS Mathematics, 6(7), 7494-7517. DOI 10.3934/math.2021438.

22. Qi, F., Zou, Q., Guo, B. N. (2019). The inverse of a triangular matrix and several identities of the Catalan numbers. Applicable Analysis and Discrete Mathematics, 13(2), 518-541. DOI 10.2298/AADM190118018Q.

23. Wang, Y., Dağl1, M. C., Liu, X. M., Qi, F. (2021). Explicit, determinantal, and recurrent formulas of generalized Eulerian polynomials. Axioms, 10(1), 37. DOI 10.3390/axioms10010037.

24. Corcino, R. B. (1999). The $(r, \beta)$-Stirling numbers. Mindanao Forum, 14, 91-99.

25. Corcino, R. B., Corcino, C. B. (2012). The Hankel transform of generalized Bell numbers and its $q$-analogue. Utilitas Mathematica, 89, 297-309.

26. Corcino, R. B., Corcino, C. B., Aldema, R. (2006). Asymptotic normality of the $(r, \beta)$-Stirling numbers. Ars Combinatoria, 81, 81-96. 